\title{
Visual Language in Visual Communication
}

\author{
Jia Wang \\ College of Art and Appareluages, Tianjin Polytechnic University, Tianjin 300160, China \\ E-mail: just_do_myself@msn.com
}

\begin{abstract}
In visual communication the design information is mainly communicated by visual language, the correct use of which is the standard of evaluation of a graphic design composition. Therefore it is necessary to understand and percept visual language properly. It will be helpful for viewers to percept the desired information from the designer as well as the significance within the work.
\end{abstract}

Keywords: Visual communication, Visual language, Motion presentation, Verbal communication, Non-verbal communication

Art, instead of being only regarded as fine art or art activity, has been involved in our daily life or even been part of everyday life. All kinds of advertisements are available everywhere. Principles and elements of design could be recognizable on commodities packing, books and magazines etc.

Visual communication, as a visual way of art design in social life, is the presentation of information by visual recognition and it is also the most commonly used way with the longest history for human beings.

\section{Conception of visual language}

In nowadays communications of mankind can be divided into two aspects: direct communication between people with languages, words, gestures and so on; indirect communication between people via objects which are very popular and typical in logo, advertising, illustration, product design and packing etc. the latter forms a different way to communicate each other. That is visual language.

Visual language is a sound set of principles and elements of design which carry meanings. Certain arrangements of words, symbols, hues, values etc, the base of design, have certain significance, which forms visual language and has impact to people. All kinds of information are carried with the movement and change of the design order, direction, illusion, psychological implication.

Visual language is an effective tool to indicate one's attitude, behavior, or even cultural preference.

\section{Application of visual language}

In communication theories, communication occurs through "verbal symbols" and "non-verbal symbols". The former mainly refers to languages and words while the latter includes music, dance, drawing, architecture and even body language such as gestures. They both are for the presentation of emotion and perception.

Samovar, an American expert in semantics, indicates that words are symbols, of course. They are not the thing itself for it is a non-acute system. People from different places and cultural background will have different perception and imagery about one thing when it is described via words. However, pictures are able to present things in a relatively clear way. In this case, visual language becomes one of the most important ways to communicate between people.

Visual language is largely recognizable due to its popularity. People from all walks of life are able to percept the significance of this kind of language in their own way. It is the designer's duty to look for the correct visual language adherence to some significance. Moreover, the language should be of creation and cultural value.

\section{Emotional presentation of visual language}

Emotion is defined as response to things and people via gestures and expression due to psychological instinct. The emotional presentation of visual language, with the fundament of visual physics and mentality, is the combination and arrangement of elements of design such as pattern, hue, words etc. Since visual language is impressively varied, it is possible to have different emotional experience.

\section{1 emotional presentation of form}

Forms can greatly be influenced by psychology. A rectangular form, for example, stays put in relation to gravity, and are not likely to tip over. A triangle with upside down suggests instability. Form itself means strong plenty. Lines communicate a feeling of delight. Rectilinear lines suggest speed, while curved lines suggest comfort, safety, familiarity. 
All these elements bring people different emotions. The combination of point, line and form suggest passion, nervousness, comfort, relaxation etc, which are the fundament of visual communication.

Objects, as material for graphic design, keep images in our convention instead of a single significance. They will have new meanings with different backgrounds. Forms and shapes can be thought of as positive or negative. In a two dimensional composition, the objects constitute the positive forms, while the background is the negative space. It is difficult to ignore the background and treat it as merely empty space. The effective placement of objects in relation to the surrounding negative space is essential for success in composition.

\section{2 emotional presentation of hue}

Color is one of the most powerful of elements. It has tremendous expressive qualities as well as forms and shapes. Understanding the uses of color is crucial to effective composition in design and the fine arts.

Hue has priority to other elements in visual design. Color illusion, as an important element, can directly suggest information which is not available via non-verbal and form symbols. Color arrangements are very powerful and have enormous impact on our responses to color. It is unsuitable to have cool color as background when advertising a warm-air device. It brings coldness to viewers who will have wrong impression for the new product. In this case, the advertisement will have no effect.

Hue, as presentation of culture, carries certain meaning even takes place of verbal symbols in certain circumstances. Non-verbal cues function via stimulating passion and emotion beyond verbal symbols. Culture significance in hue has something to do with the tradition and convention of a country and nationality. Certain colors are related to certain situations, which grow from regional culture. "Korea fashion", for example, is recognizable in traditional Korean garments. Lightness and color are combined in their design, which form strong visual impact and impression.

\section{3 emotional presentations of format and written design}

Although it must be visual when emphasizing visual communication due to our impressive convention of languages and words, we have no reason to ignore the importance of written design, especially the literalness format in printing.

A writing format in printing within visual communication is crucial to the success of a composition. Visual tendency in a writing format has great deal to do with psychological physics and mental circumstances. Variations of combination of point, line, form, pattern, hue and the way in which these elements are combined have different functions respectively.

Format design is the orderly and personally combination and arrangements of elements to carry information. It will have quality of aesthetics and economy as well as spiritual value.

Different format design, placement of patterns and selection of media avenue are used in visual language and communication. Visual tendency in the center, horizontal and vertical line on the page suggest plenty of attraction and impact with graceful symmetry, while visual tendency with round edges means stability, inside power and weak aggressiveness. Meanwhile, the impact of forms with smooth edges also has something to do with the concrete shape. Circle and triangle outline supply different inside power and specialties. Forms and shapes with uneasy edges are of impact, random and personal. Shapes with open visual format have less attention and control and more separate and aggressiveness, which occupy large space. Relatively, shapes with close form are easy to recognize. The density of writing is visual with change and variation.

Font, as a tool of graphic design, is of importance in application. Variation of font suggests different functions. Some font indicates humor, while some means reverence. Also we can recognize weakness and power in font. Human impression can be found in font. A post with a serious content, for example, is not able to carry the information of reverence and power and becomes meaningless if round font is used. A composition has strong visual effect and emotion with the arrangement of large and small font.

\section{Conclusion}

To summarize, visual language, the tool to stimulate art design, is changeable and developing instead of stationary. The communication of visual information lies not only to the accuracy and scientific objectivity but also effectiveness and discrepancy of media. It is with this emphasis that good result in visual communication is available.

\section{References}

Arnheim, Rudolf. (1998). Aesthetics design art education. Sichuan People's Press.

Aronson, E., Wilson, Timothy D. \& Akert, Robin M. (2007). Social psychology (5th ed.). China Light Industry Press.

Samovar, Larry A. \& Porter, Richard E. (2005). Communication between cultures (5th ed.). Peking University Press.

Wang, Shouzhi. (1998). A history of modern design. China Youth Press. 